\title{
A Network Pharmacology-Based Strategy for Unveiling the Mechanisms of Tripterygium Wilfordii Hook F against Diabetic Kidney Disease
}

\author{
Yuyang Wang $\mathbb{D},{ }^{1,2}$ Tongtong Liu, ${ }^{1}$ Fang Ma, ${ }^{1}$ Xiaoguang Lu, ${ }^{1}$ Huimin Mao, ${ }^{1}$ Weie Zhou, ${ }^{3}$ \\ Liping Yang, ${ }^{1}$ Ping $\mathrm{Li}\left(\mathbb{D},{ }^{3}\right.$ and Yongli Zhan ${ }^{1}{ }^{1}$ \\ ${ }^{1}$ Department of Nephrology, Guang'anmen Hospital of China Academy of Traditional Chinese Medical Sciences, \\ Beijing 100053, China \\ ${ }^{2}$ Tianjin University of Traditional Chinese Medicine, Tianjin 301617, China \\ ${ }^{3}$ Beijing Key Laboratory for Immune-Mediated Inflammatory Diseases, Institute of Clinical Medical Sciences, China- \\ Japan Friendship Hospital, Beijing 100029, China
}

Correspondence should be addressed to Ping Li; lp8675@163.com and Yongli Zhan; zhanyongli88@sina.com

Received 21 May 2020; Revised 1 October 2020; Accepted 2 November 2020; Published 20 November 2020

Academic Editor: Daniela Foti

Copyright (C) 2020 Yuyang Wang et al. This is an open access article distributed under the Creative Commons Attribution License, which permits unrestricted use, distribution, and reproduction in any medium, provided the original work is properly cited.

Background. Diabetic kidney disease (DKD) poses a major public-health burden globally. Tripterygium wilfordii Hook F (TwHF) is a widely employed herbal medicine in decreasing albuminuria among diabetic patients. However, a holistic network pharmacology strategy to investigate the active components and therapeutic mechanism underlying DKD is still unavailable. Methods. We collected TwHF ingredients and their targets by traditional Chinese Medicine databases (TCMSP). Then, we obtained DKD targets from GeneCards and OMIM and collected and analyzed TwHF-DKD common targets using the STRING database. Protein-protein interaction (PPI) network was established by Cytoscape and analyzed by MCODE plugin to get clusters. In addition, the cytoHubba software was used to identify hub genes. Finally, all the targets of clusters were subjected for Gene Ontology (GO) and Kyoto Encyclopedia of Genes and Genomes (KEGG) pathway enrichment analyses via DAVID. Results. A total of 51 active ingredients in TwHF were identified and hit by 88 potential targets related to DKD. Compounds correspond to more targets include kaempferol, beta-sitosterol, stigmasterol, and Triptoditerpenic acid B, which appeared to be high-potential compounds. Genes with higher degree including VEGFA, PTGS2, JUN, MAPK8, and HSP90AA1 are hub genes of TwHF against DKD, which are involved in inflammation, insulin resistance, and lipid homeostasis. Kaempferol and VEGFA were represented as the uppermost active ingredient and core gene of TwHF in treating DKD, respectively. DAVID results indicated that TwHF may play a role in treating DKD through AGE-RAGE signaling pathway, IL-17 signaling pathway, TNF signaling pathway, insulin resistance, and calcium signaling pathway $(P<0.05)$. Conclusion. Kaempferol and VEGFA were represented as the uppermost active ingredient and core gene of TwHF in treating DKD, respectively. The key mechanisms of TwHF against DKD might be involved in the reduction of renal inflammation by downregulating VEGFA.

\section{Introduction}

Diabetic kidney disease (DKD), a highly prevalent microvascular complication of diabetes, is characterized by thickening of the glomerular basement membrane, mesangial expansion, podocyte, and glomerular injury, leading to glomerular sclerosis and tubulointerstitial fibrosis [1]. It occurs in almost $20-50 \%$ patients with type 1 or type 2 diabetes worldwide, and even in the presence of adequate glycemic control, it remains the main cause of morbidity and mortality among diabetic patients $[2,3]$. Since those living with DKD are at higher risks of progression to cardiovascular comorbidities and end-stage kidney disease (ESKD), thus DKD is emerging as a major healthcare challenge around the globe. The clinical hallmark of DKD is described as the presence of progressive decline in renal function and persistent excretion of urinary 
albumin. Several mechanisms contribute to DKD pathogenesis through driving an overproduction of advanced glycation end products, hemodynamic changes, inflammation, oxidative stress, fibrosis, endothelial, and podocyte injury, ultimately leading to albuminuria and reduced renal function [3]. Current standard therapies to manage DKD recommend drugs controlling blood pressure and hyperglycemia, mainly including renin angiotensin aldosterone system (RAAS) inhibitors, sodium glucose cotransporter 2 (SGLT2) inhibitors, and glucagon-like peptide 1 (GLP1) receptor agonist. Despite those strategies showing promising results in DKD, many diabetic patients continue to progress to DKD and ESKD $[3,4]$. The multifactorial origin of DKD and the multiple underlying molecular mechanisms involved make it difficult to achieve successful results with one single drug. Novel therapeutic agents based on multiple targets contribute to DKD progression are therefore urgently required.

Guided by accumulative empirical experience, Chinese herbal medicine [5] acts as an effective therapy in the treatment of DKD for centuries in China and some Asian countries. Different from chemical medications, it is reported that Chinese herbal medicine [5] targets on diverse pathways to restore cellular homeostasis, which may provide a new option for the treatment of multifactorial diseases like DKD [6]. Tripterygium wilfordii Hook F (TwHF) is the most well-known drug for DKD among CHM and exerts a remarkable effect in decreasing albuminuria excretion. Evidence obtained from clinical trials suggested treatment of TwHF in combination of ARB/ACEI is more effective than $\mathrm{ARB} / \mathrm{ACEI}$ monotherapy in reduction of proteinuria for DKD patients $[7,8]$. A wide spectrum of biological activities about TwHF including anti-inflammatory, immunosuppressive, antioxidation, and antifibrosis has been demonstrated in the last decades [9-13], which may attribute to its renoprotective effects against DKD $[14,15]$. However, a satisfactory explanation of its pharmacological mechanisms and material bases related to DKD remains unavailable at the moment.

Network pharmacology is an interactive network used to study "compound-proteins/genes-disease" pathways, which own the ability of elucidating complexities for biological systems, drugs, and diseases from a systemic perspective [16, 17]. This concept shares a quite similar holistic philosophy as $\mathrm{CHM}$, which supplies a comprehensive method to systematically reveal the interactions between multicomponents, multitargets, and multipathways of the active ingredients in CHM $[18,19]$. This study is aimed at exploring the pharmacological mechanisms of TwHF for DKD employing network pharmacology approach. Our workflow was exhibited in Figure 1.

\section{Material and Methods}

2.1. TwHF Database Construction and Prediction of Target Genes. The Traditional Chinese Medicine Systems Pharmacology Database (TCMSP, http://tcmspw.com/tcmsp.php) was employed to collect the chemical compounds of TwHF, which is a unique systematic pharmacology platform of CHM that displays the associations between drugs, targets, and diseases [20]. We screened the TwHF compounds using
ADME (absorption, distribution, metabolism, and excretion) models, and the ingredients with $\mathrm{OB}$ (oral bioavailability $(\mathrm{OB})) \geq 30 \%$ and the DL (drug-likeness (DL)) $\geq 0.18$ were screened out as candidate compounds for further analysis according to a suggested criterion by the TCMSP database. Meanwhile, the target genes related to active ingredients of TwHF were gathered based on the TCMSP database. UniProt database (https://www.uniprot.org) provides access to a vast amount of sequence and functional information of proteins in a comprehensive, high-quality, and freely accessible way for scientists worldwide [21]. All obtained targets were subsequently put into UniProtKB, with the organism selected as "Homo sapiens," to search for official target names. Therefore, we collected the active compound and involved target genes of TwHF, and the details are described in Table S1. Additionally, components correspond to more targets (top 10) were imported into PubChem database (https:// pubchem.ncbi.nlm.nih.gov/) for acquiring the compound structures.

2.2. Identification of DKD Targets. The keyword "diabetic kidney disease" was used in the GeneCards (https://www .genecards.org) and Online Mendelian Inheritance in Man (OMIM, https://www.omim.org) databases to search for DKD-related targets. GeneCards is a comprehensive, authoritative human gene compendium that enables scientific community to effectively navigate concise genome, transcriptome, proteome, disease, and function data on all predicted and known human genes [22, 23]. OMIM is considered as the primary repository of comprehensive [24], freely accessible information on genes, genetic phenotypes, and gene-phenotype relationships.

\subsection{Construction and Analysis of the Protein-Protein} Interaction (PPI) Network. The intersection of the compound targets and DKD disease targets was determined with the semiprogrammed foot software $\mathrm{R}$ project $(\times 64$ 3.6.1) and taken as the candidate targets for the components of TwHF in the treatment of $\mathrm{DKD}$. A protein-protein interaction (PPI) network of overlapped target genes was built based on the STRING database (http://stringdb.org) with the organism set as "Homo sapiens" [25]. Afterwards, the target interaction information was drawn and analyzed in the visualization software Cytoscape (Version 3.7.2; https://www.cytoscape.org/). The color of the node was displayed in a gradient from red to yellow according to the descending order of the degree value, and a higher degree value node was utilized to present putative crucial targets in the PPI network. Besides, the cytoHubba software was used to select hub genes based on mixed character calculation (MCC) score, and the Molecular Complex Detection (MCODE), a plugin in the Cytoscape software, helped us to screen significant functional modules and get clusters in the PPI network according to $\mathrm{K}-$ core $=2$.

2.4. Functional Enrichment and Pathway Analysis. Gene Ontology (GO) and Kyoto Encyclopedia of Genes and Genomes (KEGG) pathway enrichment analyses for TwHF in DKD were performed using the DAVID (https://david .ncifcrf.gov) database [26]. Results with $P<0.05$ and $Q<$ 


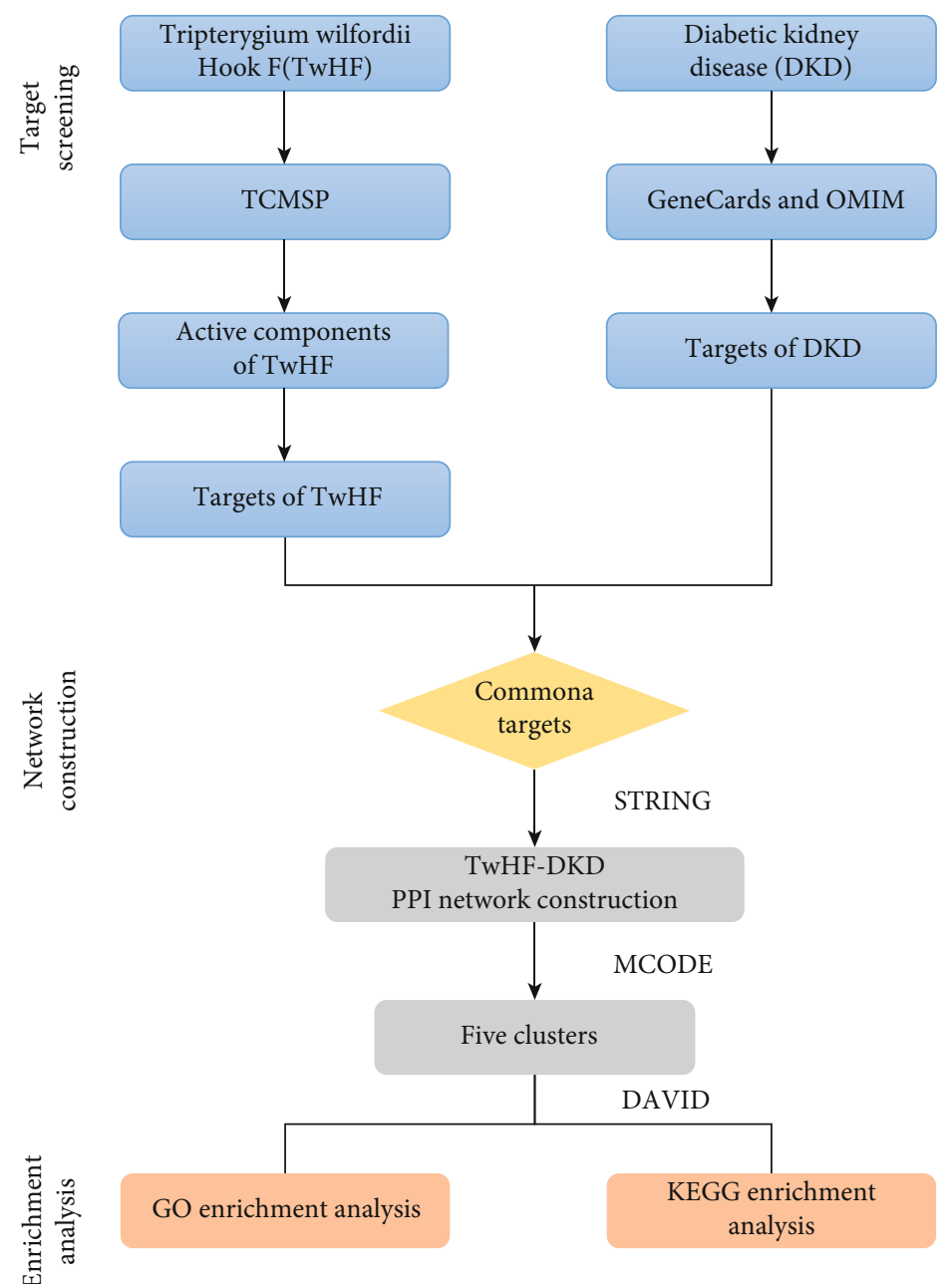

FIgURE 1: The whole framework for TwHF against DKD.

0.05 were retained for further analysis about key targets in the PPI network involved in biological processes, molecular functions, cellular components, and functional signaling pathway.

\section{Results and Discussion}

3.1. Compound-Compound Target Network. The active compound and compound targets were collected via the TCMSP database. There were 51 components in TwHF based on the ADME parameters in TCMSP of $\mathrm{OB} \geq 30 \%$ and $\mathrm{DL} \geq 0.18$ and were listed in Table 1 (detailed in Table S1 and Figure 2). Besides, a total of 93 involved targets were obtained after removing the duplicate data. We constructed a compound-compound target network in purpose of further elucidate the interactions between active ingredients of TwHF and corresponding targets, as shown in Figure 3. By mapping 51 compounds linked to 93 targets, the network consists of 126 nodes and 579 edges, in which the components calculated in yellow from TwHF, and the colorized pink circles reflect cotarget genes of TwHF-DKD, and red circles stand for component targets only. The multitarget effect of TwHF was demonstrated in this network.
Compounds correspond to more targets include kaempferol, beta-sitosterol, stigmasterol, and Triptoditerpenic acid $\mathrm{B}$, which associate with $46,39,38$, and 33 targets, respectively. The result indicated that these compounds might serve as potential ingredients for the treatment of DKD. The antidiabetic activity of kaempferol, beta-sitosterol, and stigmasterol has been reported due to their antioxidant and antiinflammatory properties while the study on Triptoditerpenic acid B was a void [27-29]. Kaempferol, a natural flavonoid, has been suggested to inhibit hyperglycemia-induced activation of inflammatory cytokines, TGF- $\beta 1$ expression, and oxidative stress in NRK-52E and human renal tubular epithelial cells [29], meanwhile, to prevent lipid accumulation and ER stress in pancreatic $\beta$ cell [30], which indicated the potential of kaempferol for treating DKD. We conducted GO and KEGG analyses of cotargets of Triptoditerpenic acid $B$ and DKD (Table S6), and the results indicated that inflammatory response might act as important mechanisms of Triptoditerpenic acid B in the treatment of DKD.

3.2. Compound Target-DKD Target Network Analysis. Using the GeneCards and OMIM databases, 11389 target genes associated with DKD were retrieved, including 11247 in 
TABLE 1: Basic information of active components in TwHF.

\begin{tabular}{|c|c|c|c|c|c|}
\hline Mol ID & Compound & MW & $\begin{array}{l}\text { OB } \\
(\%)\end{array}$ & $\mathrm{DL}$ & Targets \\
\hline MOL000422 & Kaempferol & 286.25 & 41.88 & 0.24 & 46 \\
\hline MOL000358 & Beta-sitosterol & 414.79 & 36.91 & 0.75 & 39 \\
\hline MOL000449 & Stigmasterol & 412.77 & 43.83 & 0.76 & 38 \\
\hline MOL003231 & Triptoditerpenic acid B & 328.49 & 40.02 & 0.36 & 33 \\
\hline MOL003196 & Tryptophenolide & 312.44 & 48.5 & 0.44 & 29 \\
\hline MOL003229 & Triptinin B & 314.46 & 34.73 & 0.32 & 29 \\
\hline MOL003184 & $81827-74-9$ & 342.47 & 45.42 & 0.53 & 27 \\
\hline MOL003280 & Triptonolide & 326.42 & 49.51 & 0.49 & 27 \\
\hline MOL000296 & Hederagenin & 414.79 & 36.91 & 0.75 & 26 \\
\hline MOL003217 & Isoxanthohumol & 354.43 & 56.81 & 0.39 & 26 \\
\hline MOL005828 & Nobiletin flavones & 402.43 & 61.67 & 0.52 & 25 \\
\hline MOL003248 & Triptonoterpene & 300.48 & 48.57 & 0.28 & 24 \\
\hline MOL003185 & $\begin{array}{l}\text { (1R,4aR,10aS)-5-hydroxy-1-(hydroxymethyl)-7-isopropyl-8-methoxy-1,4a-dimethyl- } \\
\text { 4,9,10,10a-tetrahydro-3H-phenanthren-2-one }\end{array}$ & 346.51 & 48.84 & 0.38 & 23 \\
\hline MOL003199 & 5,8-Dihydroxy-7-(4-hydroxy-5-methyl-coumarin-3)-coumarin & 352.31 & 61.85 & 0.54 & 22 \\
\hline MOL003283 & (2R,3R,4S)-4-(4-hydroxy-3-methoxy-phenyl)-7-methoxy-2,3-dimethylol-tetralin-6-ol & 360.44 & 66.51 & 0.39 & 22 \\
\hline
\end{tabular}

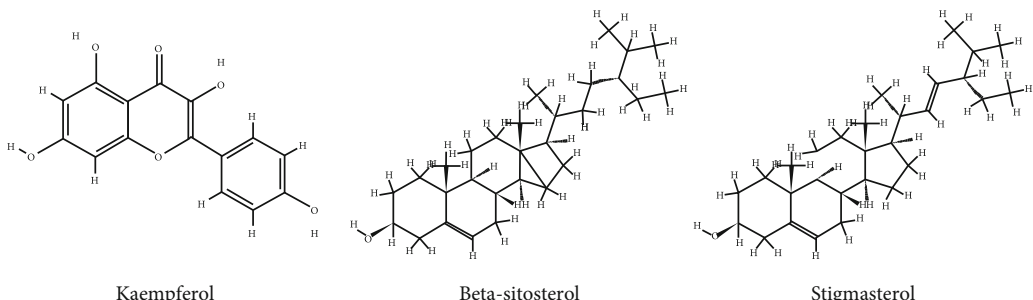

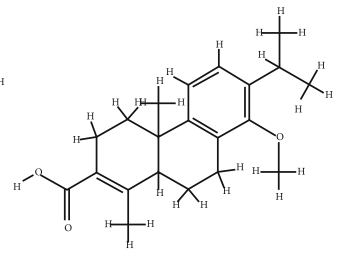

Triptoditerpenic acid B

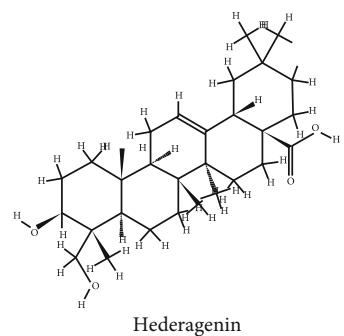

Triptonolide

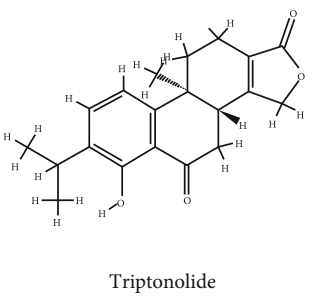

$81827-74-9$

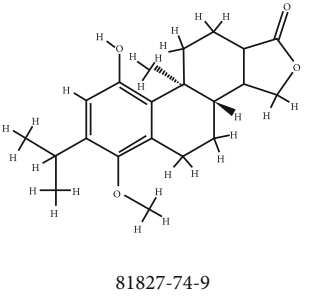

Figure 2: The chemical structure of the ten representative compounds in TwHF.

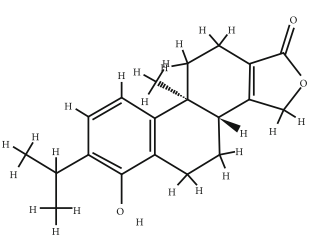

Tryptophenolide

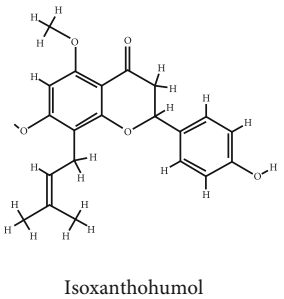

GeneCards and 142 in OMIM. A total of 11261 targets were obtained after striking out redundant entries. The details are displayed in Table S2. In total, 88 overlapping genes were determined via matching the compound-related 93 target genes with DKD-related 111261 target genes (Figure S1) and were imported into the STRING database where the PPI network was later established for analyzing the relationship between compound targets and disease targets. Almost all of them (88) among a total of 93 targets of TwHF can directly affect DKD-related targets, indicating that TwHF was promising candidates for preventing and treating DKD. As shown in Figure 4(a), this network composed of 88 nodes and 547 edges, and the average node degree is 12.4. The nodes and edges represent target genes and interactions between a pair of target genes, respectively.
The color of the node was displayed in a gradient from red to yellow according to the descending order of the degree value.

Targets with higher degrees including VEGFA, PTGS2, JUN, MAPK8, and HSP90AA1 were identified as hub genes of TwHF against DKD based on cytoHubba analysis. These genes are mostly related to chronic inflammation, insulin resistance, lipid homeostasis, oxidative stress, and fibrosis [31-34].

Vascular endothelial growth factor A (VEGFA), a predominant proangiogenic factor in the kidney, is secreted by podocytes and provides essential maintenance signals for endothelial cells, podocytes, and mesangial cells in the glomerulus [35]. Chronic hyperglycemia and excessive production of advanced glycation end products (AGEs) induce 


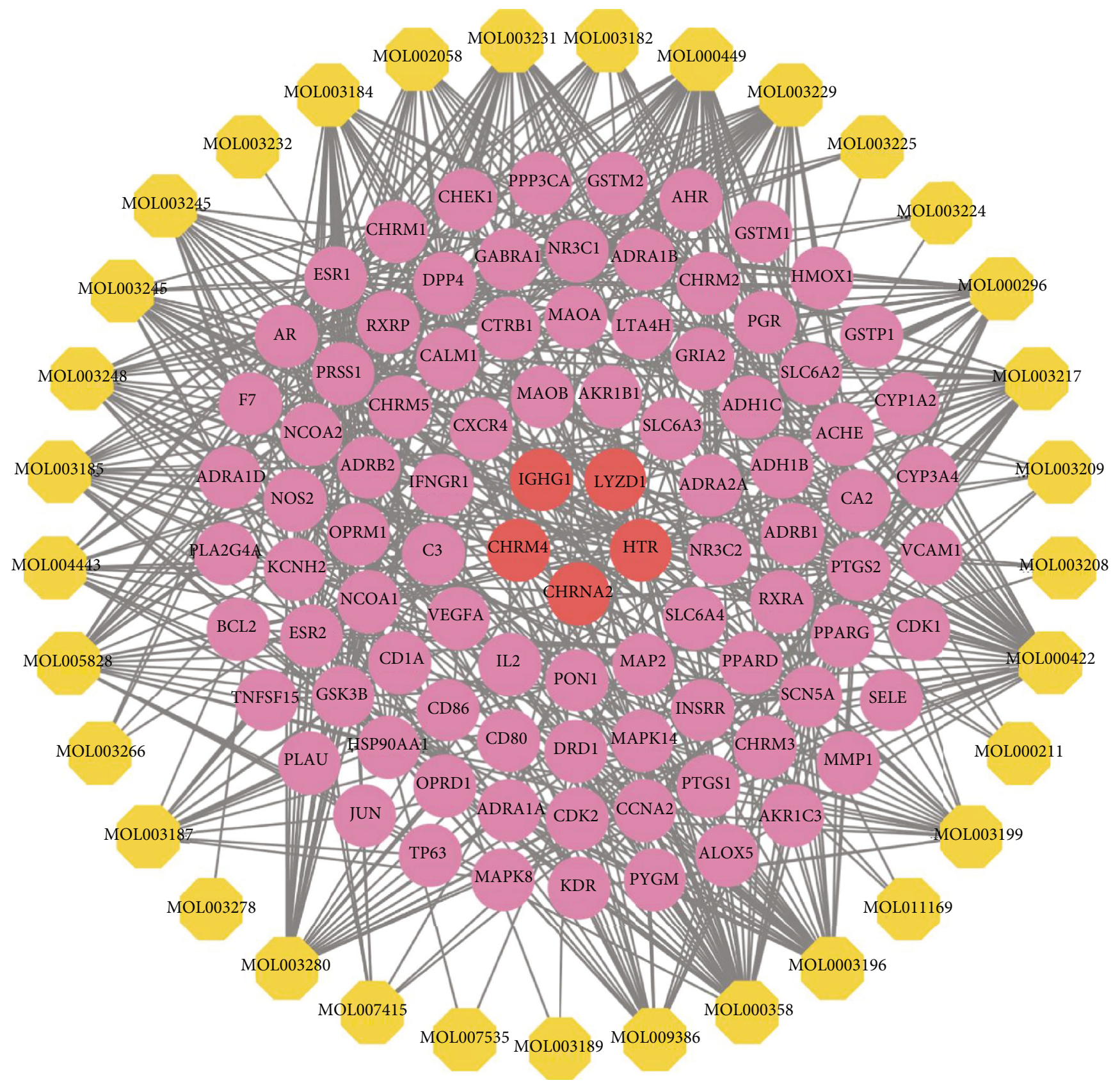

Figure 3: Compound-compound target network of targets in TwHF. The yellow hexagon stands for compounds in TwHF. The nodes represented target genes of TwHF, in which pink nodes stand for common target of TwHF-DKD, and red nodes were employed to represent compound targets not related to DKD. The lines stand for interactions between compounds and target nodes.

VEGFA synthesis and secretion, thereby stimulating abnormalities in multiple signaling pathways including inflammation, excessive ROS generation, TGF- $\beta$ and CTGF activation, and foot process effacement and ultimately leading to extracellular matrix (ECM) accumulation, GBM thickening, and albuminuria $[31,36]$. VEGFA inhibitor could lead to remission in albuminuria, glomerular hypertrophy, and endothelial activation in streptozotocin-induced diabetic mice [36]. Prostaglandin synthases (PTGS), including PTGS1 and PTGS2, are known proinflammatory cytokines and have been implicated in the pathogenesis of numerous inflammatory diseases like diabetes [32]. Both enzymes are reported to be found in pancreatic tissue, and there is evidence that PTGS2 is involved in inflammatory mediator-induced damage of pancreatic $\beta$ cells [33]. c-JUN N-terminal kinase (JNK) and p38 mitogen-activated protein kinase (p38 MAPK) are stress-activated protein kinases. Studies have shown that glomerular and tubulointerstitial p38 MAPK/JNK signaling was dramatically activated in renal biopsies from patients diagnosed with diabetes and a number of diabetic animal models $[37,38]$. Notably, activation of p38 MAPK has been reported to be strongly associated with renal inflammation and fibrosis in the settings of diabetes, while inhibition of p38 MAPK ameliorated proinflammation and 


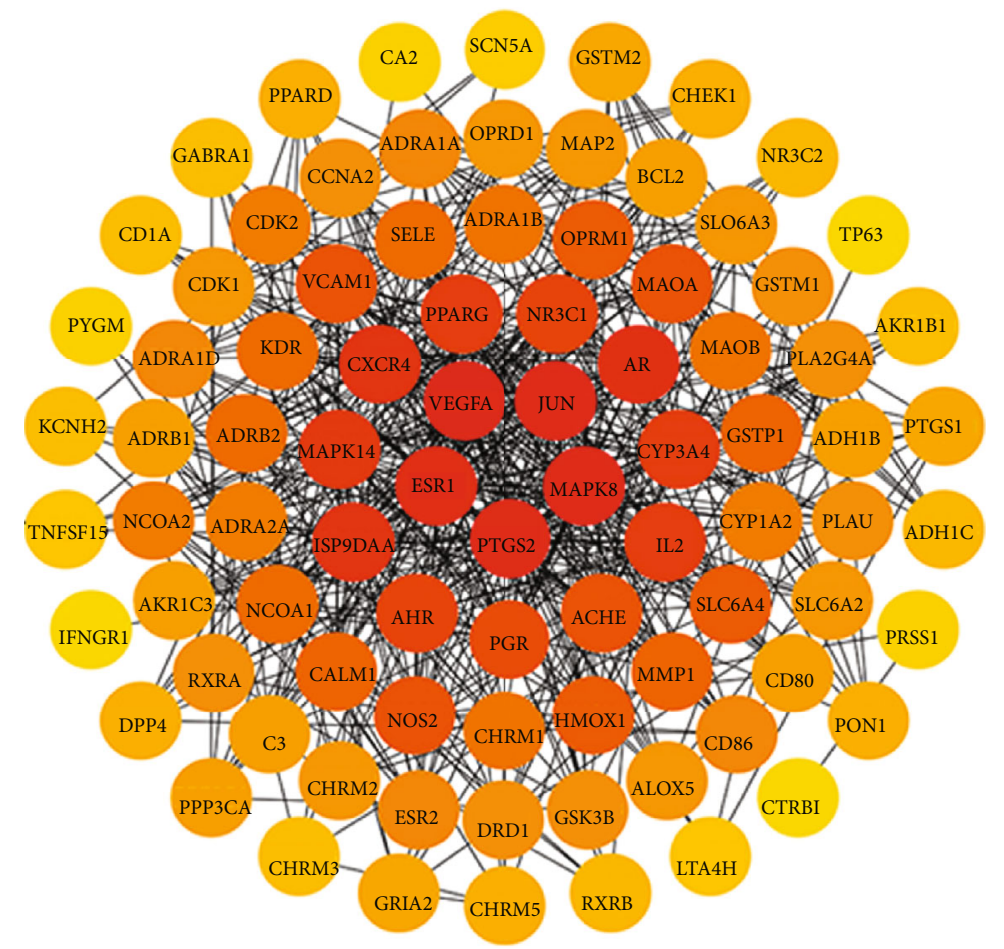

(a)

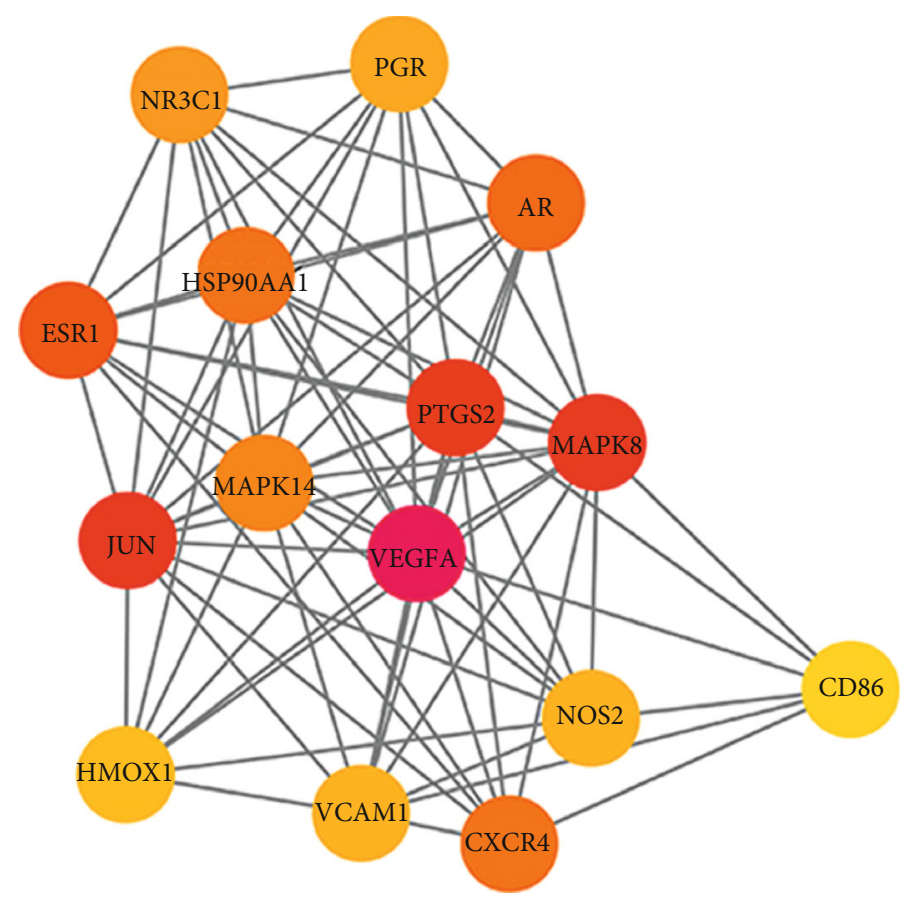

(b)

FIgURe 4: Continued. 


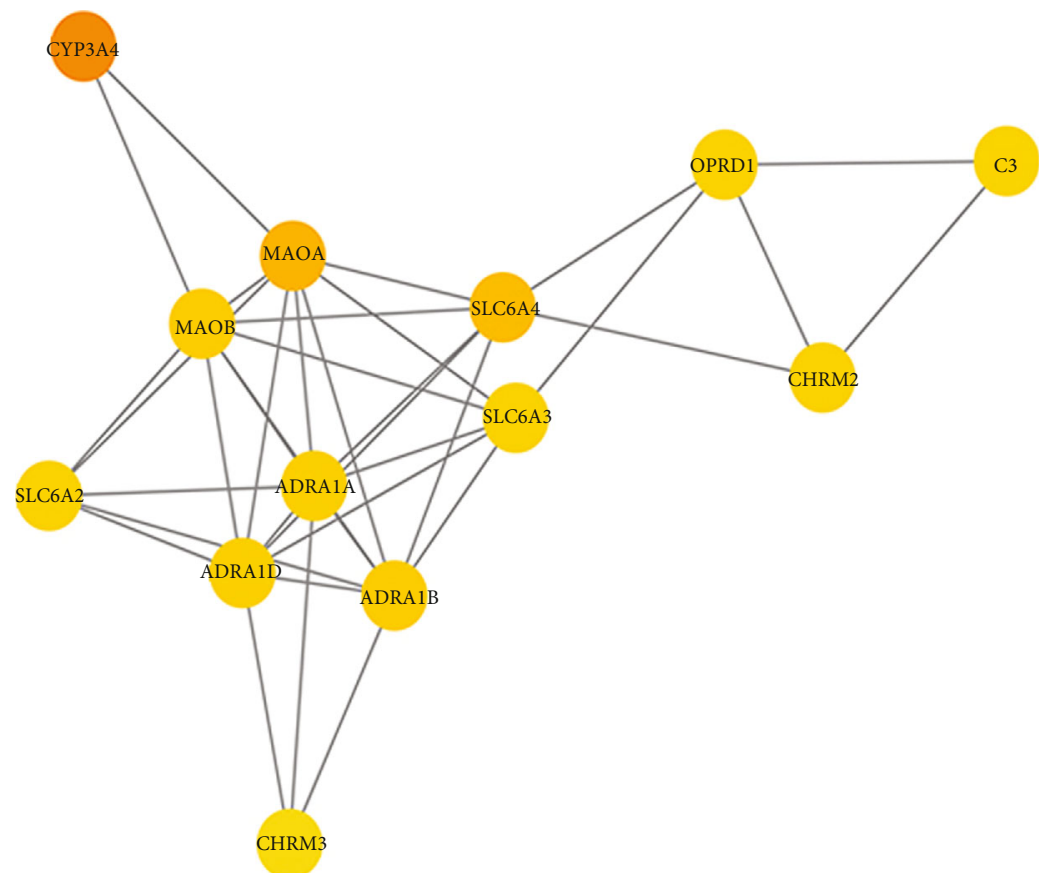

(c)
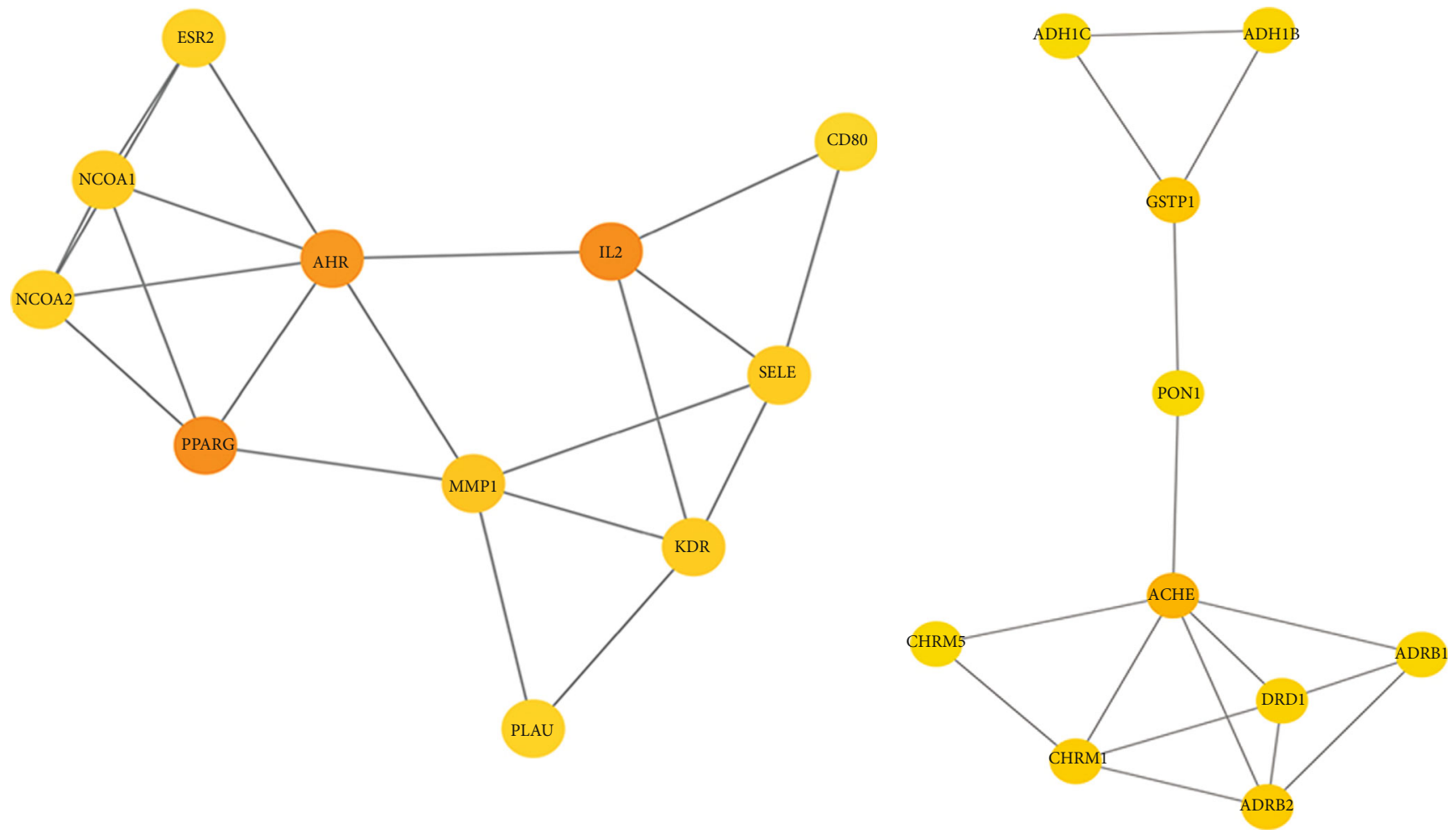

(d)

(e)

FIgUre 4: Continued. 


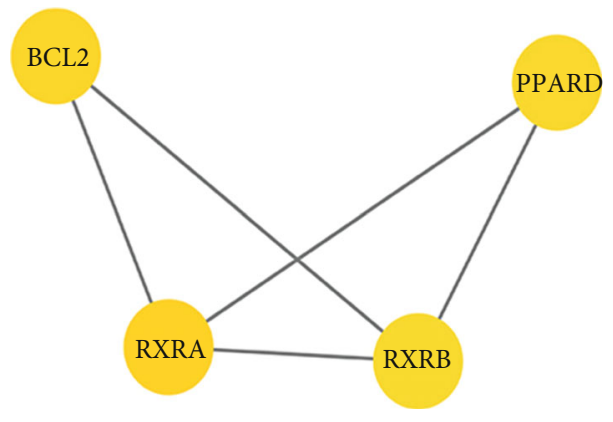

(f)

FIGURE 4: Compound-DKD PPI network and its clusters. (a) TwHF target-DKD target PPI network comprised of 88 nodes and 547 edges. The nodes stand for genes, and the lines stand for the interactions between a pair of target genes; The node color was redder as the degree value increased. (b-f) Clusters of compound-DKD PPI network. Five clusters were obtained in compound-DKD PPI network, and (b-f) stand for clusters 1-5.

TABLE 2: Cluster of compound-DKD PPI network.

\begin{tabular}{ccccc}
\hline Cluster & Score & Nodes & Edges & Genes \\
\hline 1 & 11.29 & 15 & 79 & AR, MAPK8, JUN, VEGFA, NR3C1, ESR1, MAPK14, NOS2, HSP90AA1, PGR, HMOX1, CD86, PTGS2, \\
VCAM1, and CXCR4
\end{tabular}

profibrotic responses. In addition, hyperactivation of mitogen-activated protein kinase 8 (MAPK8) plays a pivotal role in the pathogenesis of insulin resistance and obesity, while MAPK8 knockout mice presented improved adiposity and insulin sensitivity [34]. Heat shock protein C (HSP90) is predominantly expressed in podocytes and mesangial cells in the glomeruli. Despite without any changes of HSP90 expression in the renal cortex of type 2 diabetic mice and hyperglycemia incubated glomerular cells [39], HSP90 inhibition has been demonstrated to present a beneficial effect in modulating lipid homeostasis and suppressing renal inflammation as indicated by the inactivation of NF- $\kappa \mathrm{B}$ and STAT signaling pathways in DM2 mice [40].

Overall, these results indicated that the underlying mechanism of TwHF in the treatment of DKD may be involved in the reduction of excessive inflammation and protection against islets dysfunction and lipid deposition.

3.3. Cluster of Compound-DKD PPI Network. Targets in compound-DKD PPI network were analyzed by MCODE, and 5 central gene clusters were returned (Table 2, Figures 4(b)-4(f)). These clusters were subsequently imported into DAVID for GO enrichment analysis (displayed in Table S3), and numerous DKD-related biological processes were returned including the following: (1) inflammatory and immune response: response to lipopolysaccharide (GO:0032496), regulation of inflammatory response (GO:0050727), regulation of $\mathrm{T}$ helper cell differentiation (GO:0045622), regulation of leukocyte proliferation (GO:0070663), regulation of lymphocyte proliferation (GO:0050670), and regulation of B cell proliferation (GO:0030888); (2) hypoxia and oxidative stress: response to hypoxia (GO:0001666), response to decreased oxygen levels (GO:0036293), reactive oxygen species metabolic process (GO:0072593), and nitric oxide biosynthetic process (GO:0006809); (3) blood circulation and blood pressure control: vasoconstriction (GO:0042310), negative regulation of blood vessel diameter (GO:0097756), regulation of blood pressure (GO:0008217), negative regulation of systemic arterial blood pressure (GO:0003085), and positive regulation of blood circulation (GO:1903524); (4) cellular metabolic process: cAMPmediated signaling (GO:0019933), adenylate cyclasemodulating $G$ protein-coupled receptor signaling pathway (GO:0007188), cholesterol metabolic process (GO:0008203), glucose transmembrane transport (GO:1904659), and fatty acid catabolic process (GO:0009062). These biological functions of potential targets of TwHF may represent implicated mechanisms in the treatment of DKD.

3.4. Pathway of Compound-DKD PPI Network. To further reveal the potential mechanism of the TwHF on DKD, KEGG pathway enrichment analysis was conducted on the genes in the abovementioned five clusters. As shown in Table S4 and Figure 5, there are some KEGG pathways in relation to DKD were enriched to five clusters. Cluster 1 mainly enriched in AGE-RAGE signaling pathway, IL-17 signaling pathway, TNF signaling pathway, and endocrine resistance. Cluster 2 gets neuroactive ligand-receptor interaction and 


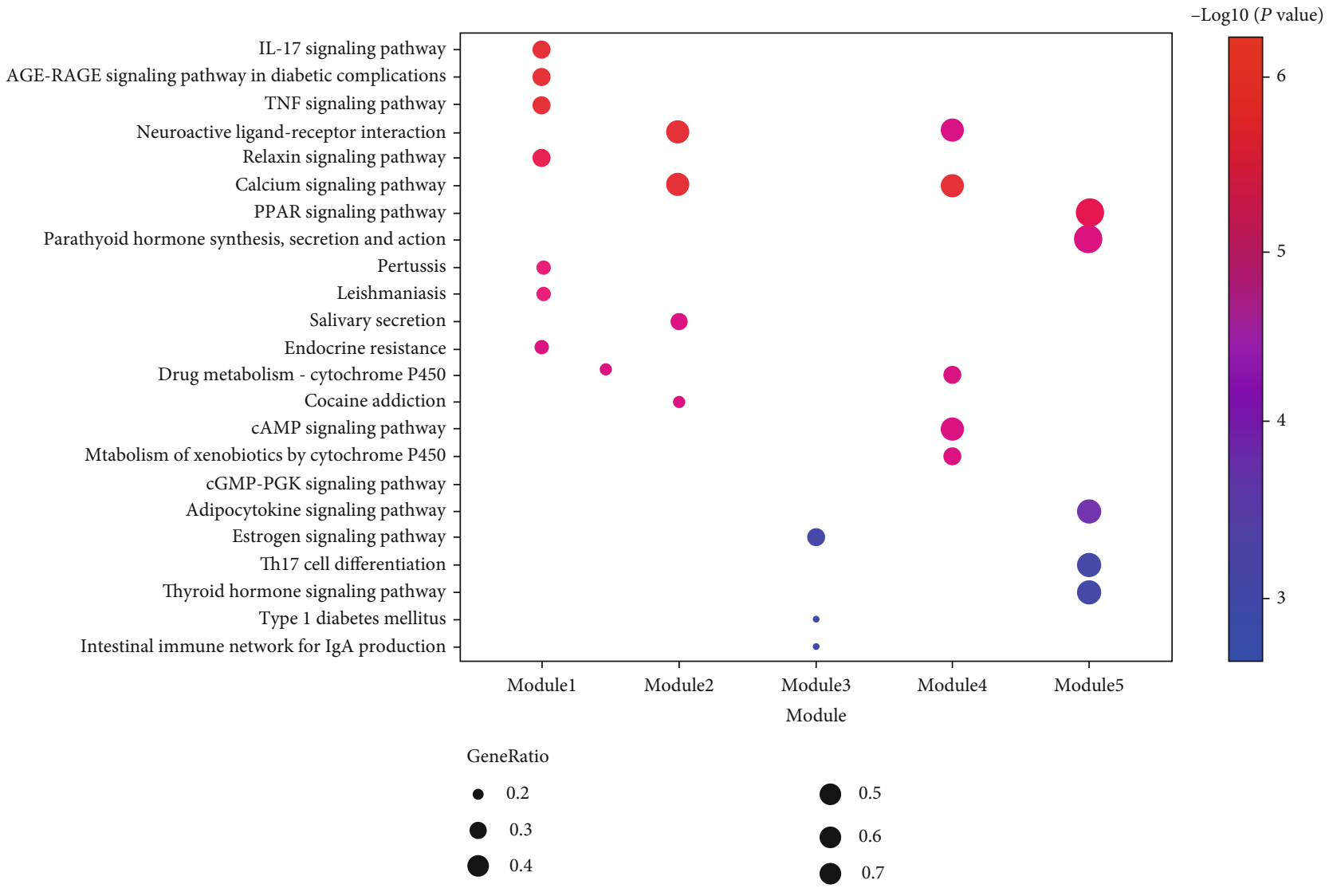

FiguRE 5: KEGG pathway analysis of 5 clusters. The color of nodes was described in a gradient from blue to red according to the descending order of the $P$ value. GeneRatio equaled to gene count enriched in pathway/total gene count enriched in the cluster.

calcium signaling pathway. Cluster 3 includes estrogen signaling pathway and type 1 diabetes mellitus. Cluster 4 gets calcium signaling pathway. Cluster 5 contains PPAR signaling pathway. Key pathways related to DKD and involved genes were described in Figure 6.

The generation and accumulation of AGEs in various tissues are highly facilitated under diabetic conditions due to persistent hyperglycemia and increased insulin resistance. The kidney acts as a crucial target for AGE-mediated impairment as well as a contributor to increasing AGE that results from a decrease in renal function, by the clearance of AGE [41]. Interaction of AGEs with the receptor for AGEs (RAGE) provokes deleterious intracellular signaling cascades, activating oxidative stress, inflammatory, and fibrotic response in diabetic kidney tissues, ultimately leading to renal dysfunction [42]. Increase in AGE-RAGE interaction under diabetic conditions activates renal VEGFA expression among various in vitro and in vivo experimental models and further aggravates the release of inflammatory factors [31]. Treatment with RAGE-aptamer to block the binding of AGEs to RAGE resulted in decreased ROS production, albuminuria, and extracellular matrix accumulation, accompanying with increased NADPH oxidase activity in kidney tissues from diabetic rats [43]. Consistently, RAGE knockout in streptozotocin-induced diabetic mice showed significantly remission in mesangial expansion or glomerular basement membrane thickening, while mice administered with AGE- albumin developed NF- $\kappa$ B activation, upregulated collagen IV, and glomerulosclerosis in kidney tissues [44]. In addition, studies suggest that AGE-RAGE axis stimulates renal inflammation through $\mathrm{NF}-\kappa \mathrm{B}$ signaling pathway and the release of central proinflammatory cytokine TNF- $\alpha$, and the interaction of AGE-RAGE and TNF signaling pathway could induce the excessive generation of superoxide in diabetes [45].

Chronic inflammation has a pivotal role in the development and progression of DKD. Tumor necrosis factor (TNF) functions as a critical cytokine in regulating a wide range of intracellular signal pathways including inflammation and immunity, which has been actively implicated in the renal pathophysiology and activated mainly by NF- $\kappa \mathrm{B}$ signaling pathway and MAPK cascade [46]. There is an increasing evidence that TNF- $\alpha$ generated from renal cells and infiltrating macrophages has shown the ability to modulate several mechanisms involved in the DKD development and progression, including promoting permeability of the glomerular basement membrane and glomerular vasoconstriction, decreasing intraglomerular blood flow [47]. Recent findings from clinical specimens with DKD demonstrated that TNF gene expression of renal tubule was associated with increased fibrosis and reduced GFR [48], and there is a close correlation between a decline in renal function and circulating TNF- $\alpha$ and its receptor (TNFR) [49]. Of note, it has been reported that multiglycoside of TwHF could inhibit the activation of p38 MAPK and NF- $\kappa \mathrm{B}$ signaling and suppress 

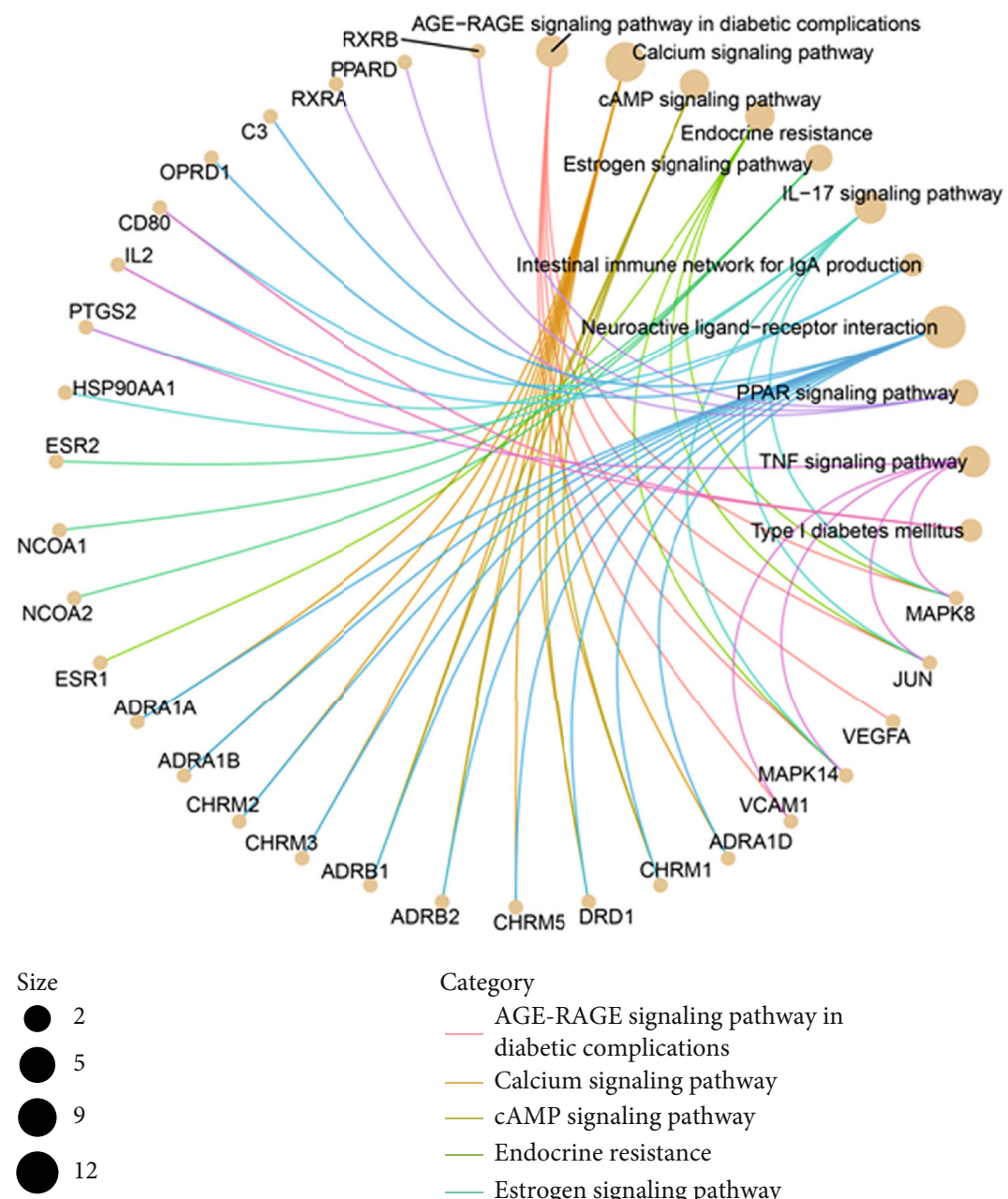

Category

AGE-RAGE signaling pathway in
diabetic complications
Calcium signaling pathway
cAMP signaling pathway
Endocrine resistance
Estrogen signaling pathway
IL-17 signaling pathway
Intestinal immune network for IgA production
Neuroactive ligand-receptor interaction
PPAR signaling pathway
TNF signaling pathway
Type 1 diabetes mellitus

FIGURE 6: The potential key signaling pathways and involved genes of TwHF for treating DKD.

overexpression of proinflammatory cytokines (TNF- $\alpha$, IL$1 \beta$, and TGF- $\beta 1$ ) in kidney, which may contribute to the improvement in glomerulosclerosis within diabetic rats [13]. Another study revealed that the intervention of triptolide, a major active component of TwHF, alleviated DKD through maintaining Th1/Th2 cell balance accompanied by downregulated IFN- $\gamma$ and TNF- $\alpha$ and upregulated IL- 4 and IL-10 [50]. Furthermore, Ma et al. confirmed that IL-17 deficient mice developed a reduction in albuminuria and an improvement in glomerular damage and fibrosis under diabetic setting, implying the contributing role of IL-17 signaling in the pathogenesis of DKD [51]. The inhibitory effect of TwHF and its extracts on IL-17 has been identified in autoimmune diseases including psoriasis, ankylosing spondylitis, and rheumatoid arthritis [52-54], but not in DKD yet.

Emerging evidence suggests that calcium signaling pathway appears to be vital in the development of DKD. It has shown that the function of native kidney cells could be controlled by intracellular $\mathrm{Ca} 2+$ signaling [55]. High glucose-induced $\mathrm{Ca}^{2+}$ alternation in mesangial cells enhanced the deposition of extracellular matrix proteins and mesangial expansion, thereby leading to glomerular injury [56], and the albumin endocytosis in proximal tubular epithelial cells was $\mathrm{Ca}^{2+}$-dependent [56]. As for podocyte, studies have revealed that calcium influx in response to angiotensin II, ROS, and other factors under diabetic conditions resulted in podocyte hypertrophy and foot process effacement, which may contribute to albuminuria and progression to DKD [57]. Additionally, increased intracellular $\mathrm{Ca} 2+$ levels promoted LKB1-AMPK/PPAR $\alpha$ pathway in high glucose-treated glomerular endothelial cells and podocytes, thus attenuating oxidative stress and apoptosis [58].

Based on the degree value of each gene in compound target-DKD target network, VEGFA was considered as the core gene of TwHF against DKD. The most potent angiogenic factor, VEGFA, is a major contributor to the development of new vessels and is also identified as a 
proinflammatory cytokine [59]. Data obtained from BTBR $\mathrm{ob} / \mathrm{ob}$ mice, streptozotocin-induced diabetic mice, and patients with DKD has demonstrated that VEGFA was upregulated under diabetic environment $[36,60,61]$, and increased VEGFA was closely related to a variety of pathological abnormalities in DKD such as glomerular hypertrophy, foot process effacement, fibrosis, and albuminuria [31], implying the crucial role of VEGFA in the pathogenesis of DKD. There is a significant relationship between VEGFA and kidney inflammation in the settings of diabetes. Elevated serum VEGFA levels in DKD patients was associated with enhanced expression of inflammatory biomarkers [61], while pharmacologic inhibition of VEGFA attenuated kidney pathological damage and albuminuria through suppressing endothelial activation and reducing glomerular macrophage infiltration and TNF- $\alpha$ expression [36]. Lavoz et al. found that blockage of VEGFR2, the receptor of VEGFA, has shown beneficial effects in treating DKD by downregulating the expression of proinflammatory mediators such as MCP-1, IL-6, and IL-17a, thus improving tubulointerstitial inflammation and ultimately reversing diabetic kidney impairment [60]. Otherwise, VEGFA expression in adipose tissue has been reported to be correlated with the development of obesity and insulin resistance [59]. Therefore, the key mechanisms of TwHF against DKD might be to rugulate VEGFA overexpression and thereby suppress a cascade of proinflammatory factors and intracellular signaling, resulting in the remission of kidney damage under diabetic conditions. Studies have revealed that triptolide, a diterpenoid triepoxide from TwHF, might exert antiangiogenic effect on rheumatoid arthritis via inhibiting VEGF, TNF- $\alpha$, and IL-17 [54], and further experiment needs to be conducted to investigate the specific pharmacological effect of TwHF on DKD.

DKD poses a major public-health burden globally attributed to the dramatically increasing prevalence of diabetes. Despite great efforts in looking for pharmacological strategies to control the disease based on the one-target one-drug paradigm, the incidence of ESRD continues to rise; thus, multiple component-multiple target pattern receives a large amount of attention recently. Tripterygium has been widely used among Chinese practitioners for thousands of years in treating DKD and exhibits remarkable effects in reducing albuminuria excretion. Clinical trials have confirmed that TwHF and its extracts are effective in treating DKD, including decreasing proteinuria and decrease in eGFR [62]. At the same time, our research indicated that TwHF might modulate lipid homeostasis and insulin resistance under diabetic environment, which provided a novel explanation for elucidating the potential mechanisms of TwHF on DKD, but our hypotheses need further experimental validation. Notably, adverse events related to TwHF treatment including liver toxicity, infertility, and hematopoietic disorders have been reported in recent years [63] and received increasingly attention. Diterpenoids, alkaloids, and triterpenoids are the major toxic components of Tripterygium [64]. We analyzed top 20 compounds of TwHF against DKD according to the Comparative Toxicogenomics Database and found that there are 6 diterpenoids, 1 triterpenoid, and 0 alkaloids. However, the high-potential components kaempferol, beta-sitosterol, and stigmasterol in the treatment of DKD belong to flavonoids and phytosterols, respectively. Therefore, our study helps to identify the active components of TwHF against DKD as well as may be regarded as a good beginning point for designing new drugs with fewer side effects.

\section{Conclusion}

Our research is the first report to explain the active ingredients and mechanisms of TwHF against DKD using network pharmacology. A total of 51 active ingredients in TwHF were screened and hit by 88 potential targets related to $\mathrm{DKD}$, and kaempferol and VEGFA were represented as the uppermost active ingredient and core gene of TwHF in treating DKD, respectively. The mechanisms of TwHF against DKD were associated with 5 functional clusters, and the key mechanisms of TwHF against DKD might be involved in the reduction of renal inflammation by downregulating VEGFA. Although the present study provided a methodological exploration for identifying potential active compound and pharmacological mechanism of TwHF on DKD, in vivo and in vitro experimental validation is required in the next step to support our findings.

\section{Data Availability}

The data used to support the findings of this study are available from the corresponding author upon request.

\section{Conflicts of Interest}

The authors declare that there is no conflict of interests regarding the publication of this paper.

\section{Acknowledgments}

This review was supported by grants from the National Natural Science Foundation of China (81804087; 81620108031) and the excellent scientific and technological youth program of China Academy of Chinese Medical Sciences Fund (ZZ13YQ-031).

\section{Supplementary Materials}

Table S1: Compound database of Tripterygium. Table S2: the GO and KEGG analyses on Triptoditerpenic acid B-DKD cotargets. Table S3: compound-DKD cotargets. Table S4: the GO enrichment analysis based on compound-DKD PPI network. Table S5: the KEGG pathway analysis based on compound-DKD PPI network. Table S6: the GO and KEGG analyses on Triptoditerpenic acid B and DKD cotargets. Table S7: the GO and KEGG analyses based on compoundDKD PPI network using WebGestalt. Figure S1: matching of target genes between DKD and TwHF. (Supplementary materials)

\section{References}

[1] H. J. Anders, T. B. Huber, B. Isermann, and M. Schiffer, "CKD in diabetes: diabetic kidney disease versus nondiabetic kidney 
disease," Nature Reviews. Nephrology, vol. 14, no. 6, pp. 361$377,2018$.

[2] N. M. Selby and M. W. Taal, "An updated overview of diabetic nephropathy: Diagnosis, prognosis, treatment goals and latest guidelines," Diabetes, Obesity and Metabolism, vol. 22, no. S1, pp. 3-15, 2020.

[3] J. Barrera-Chimal and F. Jaisser, "Pathophysiologic mechanisms in diabetic kidney disease: A focus on current and future therapeutic targets," Diabetes, Obesity and Metabolism, vol. 22, no. S1, pp. 16-31, 2020.

[4] M. Kato and R. Natarajan, "Epigenetics and epigenomics in diabetic kidney disease and metabolic memory," Nature Reviews. Nephrology, vol. 15, no. 6, pp. 327-345, 2019.

[5] T. Wendt, N. Tanji, J. Guo et al., "Glucose, glycation, and RAGE: implications for amplification of cellular dysfunction in diabetic nephropathy," J Am Soc Nephrol, vol. 14, no. 5, pp. 1383-1395, 2003.

[6] M. Lyu, Z. Zhou, X. Wang et al., "Network PharmacologyGuided Development of a Novel Integrative Regimen to Prevent Acute Graft-vs.-Host Disease," Frontiers in Pharmacology, vol. 9, 2018.

[7] C. Xiong, L. Li, W. Bo et al., "Evaluation of the efficacy and safety of TWHF in diabetic nephropathy patients with overt proteinuria and normal eGFR," Journal of the Formosan Medical Association, vol. 119, no. 3, pp. 685-692, 2020.

[8] D. Ren, C. Zuo, and G. Xu, "Clinical efficacy and safety of Tripterygium wilfordii Hook in the treatment of diabetic kidney disease stage IV," Medicine, vol. 98, no. 11, p. e14604, 2019.

[9] D. Zhao, S. Li, T. Liao et al., "Triptolide inhibits donor-specific antibody production and attenuates mixed antibody-mediated renal allograft injury," American Journal of Transplantation, vol. 18, no. 5, pp. 1083-1095, 2018.

[10] M. Xue, Y. Cheng, F. Han et al., “Triptolide attenuates renal tubular epithelial-mesenchymal transition via the MiR-1885p-mediated PI3K/AKT pathway in diabetic kidney disease," International Journal of Biological Sciences, vol. 14, no. 11, pp. 1545-1557, 2018.

[11] F. Han, S. Wang, Y. Chang et al., “Triptolide prevents extracellular matrix accumulation in experimental diabetic kidney disease by targeting microRNA-137/Notch1 pathway," Journal of Cellular Physiology, vol. 233, no. 3, pp. 22252237, 2018.

[12] X. G. Dong, Z. M. An, Y. Guo, J. L. Zhou, and T. Qin, "Effect of triptolide on expression of oxidative carbonyl protein in renal cortex of rats with diabetic nephropathy," Journal of Huazhong University of Science and Technology. Medical Sciences, vol. 37, no. 1, pp. 25-29, 2017.

[13] W. Wu, J. J. Yang, H. M. Yang et al., "Multi-glycoside of Tripterygium wilfordii Hook. f. attenuates glomerulosclerosis in a rat model of diabetic nephropathy by exerting antimicroinflammatory effects without affecting hyperglycemia," International Journal of Molecular Medicine, vol. 40, no. 3, pp. 721-730, 2017.

[14] W.-j. Huang, W.-j. Liu, Y.-h. Xiao et al., "Tripterygium and its extracts for diabetic nephropathy: Efficacy and pharmacological mechanisms," Biomed Pharmacother, vol. 121, no. 109599, p. 109599, 2020.

[15] X. Liang, B. Chen, P. Wang et al., "Triptolide potentiates the cytoskeleton-stabilizing activity of cyclosporine A in glomerular podocytes via a GSK3 $\beta$ dependent mechanism," American
Journal of Translational Research, vol. 12, no. 3, pp. 800-812, 2020.

[16] A. L. Hopkins, "Network pharmacology," Nature Biotechnology, vol. 25, no. 10, pp. 1110-1111, 2007.

[17] S. Li, "Exploring traditional chinese medicine by a novel therapeutic concept of network target," Chinese Journal of Integrative Medicine, vol. 22, no. 9, pp. 647-652, 2016.

[18] T. Suo, J. Liu, X. Chen et al., "Combining Chemical Profiling and Network Analysis to Investigate the Pharmacology of Complex Prescriptions in Traditional Chinese Medicine," Scientific Reports, vol. 7, no. 1, 2017.

[19] R. Zhang, X. Zhu, H. Bai, and K. Ning, "Network Pharmacology Databases for Traditional Chinese Medicine: Review and Assessment," Frontiers in Pharmacology, vol. 10, 2019.

[20] J. Ru, P. Li, J. Wang et al., "TCMSP: a database of systems pharmacology for drug discovery from herbal medicines," Journal of Cheminformatics, vol. 6, no. 1, 2014.

[21] The UniProt Consortium, "UniProt: the universal protein knowledgebase," Nucleic Acids Research, vol. 45, no. D1, pp. D158-D169, 2017.

[22] S. Fishilevich, S. Zimmerman, A. Kohn et al., "Genic insights from integrated human proteomics in GeneCards," Database, vol. 2016, no. 2016, article baw030.

[23] M. Safran, I. Dalah, J. Alexander et al., "GeneCards Version 3: the human gene integrator," Database, vol. 2010, article baq020, 2010.

[24] J. S. Amberger and A. Hamosh, "Searching Online Mendelian Inheritance in Man (OMIM): A Knowledgebase of Human Genes and Genetic Phenotypes," Current Protocols in Bioinformatics, vol. 58, no. 1 .

[25] D. Szklarczyk, A. L. Gable, D. Lyon et al., "STRING v11: protein-protein association networks with increased coverage, supporting functional discovery in genome-wide experimental datasets," Nucleic Acids Research, vol. 47, no. D1, pp. D607d613, 2019.

[26] W. Hu, W. Fu, X. Wei, Y. Yang, C. Lu, and Z. Liu, "A Network Pharmacology Study on the Active Ingredients and Potential Targets ofTripterygium wilfordiiHook for Treatment of Rheumatoid Arthritis," Evidence-Based Complementary and Alternative Medicine, vol. 2019, Article ID 5276865, 15 pages, 2019.

[27] O. A. Oyebode, O. L. Erukainure, C. I. Chukwuma, C. U. Ibeji, N. A. Koorbanally, and S. Islam, "Boerhaavia diffusa inhibits key enzymes linked to type 2 diabetes in vitro and in silico; and modulates abdominal glucose absorption and muscle glucose uptake ex vivo," Biomedicine \& Pharmacotherapy, vol. 106, pp. 1116-1125, 2018.

[28] B. Sudhamalla, M. Gokara, N. Ahalawat, D. G. Amooru, and R. Subramanyam, "Molecular dynamics simulation and binding studies of beta-sitosterol with human serum albumin and its biological relevance," The Journal of Physical Chemistry. B, vol. 114, no. 27, pp. 9054-9062, 2010.

[29] D. Sharma, P. Gondaliya, V. Tiwari, and K. Kalia, "Kaempferol attenuates diabetic nephropathy by inhibiting RhoA/Rhokinase mediated inflammatory signalling," Biomedicine of Pharmacotherapy, vol. 109, pp. 1610-1619, 2019.

[30] R. Varshney, R. Varshney, R. Mishra, S. Gupta, D. Sircar, and P. Roy, "Kaempferol alleviates palmitic acid-induced lipid stores, endoplasmic reticulum stress and pancreatic $\beta$-cell dysfunction through AMPK/mTOR-mediated lipophagy," 
The Journal of Nutritional Biochemistry, vol. 57, pp. 212227, 2018.

[31] A. Tufro and D. Veron, "VEGF and podocytes in diabetic nephropathy," Seminars in Nephrology, vol. 32, no. 4, pp. 385-393, 2012.

[32] Y. Wang, Y. Liu, M. Zhang et al., "Inhibition of PTGS1 promotes osteogenic differentiation of adipose-derived stem cells by suppressing NF-kB signaling," Stem Cell Research \& Therapy, vol. 10, no. 1, p. 57, 2019.

[33] A. Vennemann, A. Gerstner, N. Kern et al., "PTGS-2PTGER2/4 signaling pathway partially protects from diabetogenic toxicity of streptozotocin in mice," Diabetes, vol. 61, no. 7, pp. 1879-1887, 2012.

[34] H. Wu, Y. Wang, W. Li et al., "Deficiency of mitophagy receptor FUNDC1 impairs mitochondrial quality and aggravates dietary-induced obesity and metabolic syndrome," Autophagy, vol. 15, no. 11, pp. 1882-1898, 2019.

[35] K. L. Onions, M. Gamez, N. R. Buckner et al., "VEGFC reduces glomerular albumin permeability and protects against alterations in VEGF receptor expression in diabetic nephropathy," Diabetes, vol. 68, no. 1, pp. 172-187, 2018.

[36] P. Bus, M. Scharpfenecker, P. Van Der Wilk, R. Wolterbeek, J. A. Bruijn, and H. J. Baelde, "The VEGF-A inhibitor sFLT-1 improves renal function by reducing endothelial activation and inflammation in a mouse model of type 1 diabetes," Diabetologia, vol. 60, no. 9, pp. 1813-1821, 2017.

[37] A. K. H. Lim and G. H. Tesch, "Inflammation in diabetic nephropathy," Mediators of Inflammation, vol. 2012, Article ID 146154, 12 pages, 2012.

[38] G. Liang, L. Song, Z. Chen et al., "Fibroblast growth factor 1 ameliorates diabetic nephropathy by an anti-inflammatory mechanism," Kidney International, vol. 93, no. 1, pp. 95-109, 2018.

[39] S. Bellini, F. Barutta, R. Mastrocola, L. Imperatore, G. Bruno, and G. Gruden, "Heat shock proteins in vascular diabetic complications: review and future perspective," International Journal of Molecular Sciences, vol. 18, no. 12, p. 2709, 2017.

[40] I. Lazaro, A. Oguiza, C. Recio et al., "Targeting HSP90 ameliorates nephropathy and atherosclerosis through suppression of NF- $\kappa$ B and STAT signaling pathways in diabetic mice," Diabetes, vol. 64, no. 10, pp. 3600-3613, 2015.

[41] W. H. Yiu, D. W. L. Wong, H. J. Wu et al., "Kallistatin protects against diabetic nephropathy in $\mathrm{db} / \mathrm{db}$ mice by suppressing AGE-RAGE-induced oxidative stress," Kidney International, vol. 89, no. 2, pp. 386-398, 2016.

[42] D. Sanajou, A. G. Haghjo, H. Argani, and S. Aslani, "AGERAGE axis blockade in diabetic nephropathy: Current status and future directions," European Journal of Pharmacology, vol. 833, pp. 158-164, 2018.

[43] T. Matsui, Y. Higashimoto, Y. Nishino, N. Nakamura, K. Fukami, and S. I. Yamagishi, "RAGE-aptamer blocks the development and progression of experimental diabetic nephropathy," Diabetes, vol. 66, no. 6, pp. 1683-1695, 2017.

[44] A. L. Y. Tan, J. M. Forbes, and M. E. Cooper, "AGE, RAGE, and ROS in diabetic nephropathy," Seminars in Nephrology, vol. 27, no. 2, pp. 130-143, 2007.

[45] D. He, J.-h. Huang, Z.-y. Zhang et al., “A Network Pharmacology-Based Strategy For Predicting Active Ingredients And Potential Targets Of LiuWei DiHuang Pill In Treating Type 2 Diabetes Mellitus," Drug Design, Development and Therapy, vol. Volume 13, pp. 3989-4005, 2019.
[46] S. Bernardi, R. Voltan, E. Rimondi et al., "TRAIL, OPG, and TWEAK in kidney disease: biomarkers or therapeutic targets?," Clinical Science (London, England), vol. 133, no. 10, pp. 1145-1166, 2019.

[47] T. Gohda and Y. Tomino, "Novel biomarkers for the progression of diabetic nephropathy: soluble TNF receptors," Current Diabetes Reports, vol. 13, no. 4, pp. 560-566, 2013.

[48] J. Park, Y. Guan, X. Sheng et al., "Functional methylome analysis of human diabetic kidney disease," JCI Insight, vol. 4, no. 11, 2019.

[49] T. Gohda, Y. Nishizaki, M. Murakoshi et al., "Clinical predictive biomarkers for normoalbuminuric diabetic kidney disease," Diabetes Research and Clinical Practice, vol. 141, pp. 62-68, 2018.

[50] H. Guo, C. Pan, B. Chang et al., "Triptolide improves diabetic nephropathy by regulating Th cell balance and macrophage infiltration in rat models of diabetic nephropathy," Experimental and Clinical Endocrinology \& Diabetes, vol. 124, no. 6, pp. 389-398, 2016.

[51] J. Ma, Y. J. Li, X. Chen, T. Kwan, S. J. Chadban, and H. Wu, "Interleukin 17A promotes diabetic kidney injury," Scientific Reports, vol. 9, no. 1, 2019.

[52] S. Meng, L. Sun, L. Wang et al., "Loading of water-insoluble celastrol into niosome hydrogels for improved topical permeation and anti-psoriasis activity," Colloids and Surfaces B: Biointerfaces, vol. 182, p. 110352, 2019.

[53] W. E. I. JI, Y. A. J. U. N. CHEN, X. I. A. ZHAO et al., "Beneficial effects of tripterygium glycosides tablet on biomarkers in patients with ankylosing spondylitis," Molecular Medicine Reports, vol. 12, no. 1, pp. 684-690, 2015.

[54] X. Kong, Y. Zhang, C. Liu et al., "Anti-angiogenic effect of triptolide in rheumatoid arthritis by targeting angiogenic cascade," PLoS ONE, vol. 8, no. 10, p. e77513, 2013.

[55] S. Chaudhari and R. Ma, "Store-operated calcium entry and diabetic complications," Experimental Biology and Medicine, vol. 241, no. 4, pp. 343-352, 2015.

[56] B. Zeng, G.-L. Chen, E. Garcia-Vaz et al., "ORAI channels are critical for receptor-mediated endocytosis of albumin," Nature Communications, vol. 8, no. 1, p. 1920, 2017.

[57] J. S. Kang, S. J. Lee, J.-H. Lee et al., “Angiotensin II-mediated MYH9 downregulation causes structural and functional podocyte injury in diabetic kidney disease," Scientific Reports, vol. 9, no. 1, p. 7679, 2019.

[58] Y. Kim, J. H. Lim, M. Y. Kim et al., "The adiponectin receptor agonist AdipoRon ameliorates diabetic nephropathy in a model of type 2 diabetes," J Am Soc Nephrol, vol. 29, no. 4, pp. 1108-1127, 2018.

[59] L. A. Fatima, R. S. Campello, R. de Souza Santos et al., "Estrogen receptor 1 (ESR1) regulates VEGFA in adipose tissue," Scientific Reports, vol. 7, no. 1, p. 16716, 2017.

[60] C. Lavoz, R. R. Rodrigues-Diez, A. Plaza et al., "VEGFR2 Blockade Improves Renal Damage in an Experimental Model of Type 2 Diabetic Nephropathy," Journal of Clinical Medicine, vol. 9, no. 2, p. 302, 2020.

[61] M. Hanefeld, D. Appelt, K. Engelmann et al., "Serum and plasma levels of vascular endothelial growth factors in relation to quality of glucose control, biomarkers of inflammation, and diabetic nephropathy," Hormone and Metabolic Research, vol. 48, no. 8, pp. 529-534, 2016.

[62] Y. Ge, H. Xie, S. Li et al., "Treatment of diabetic nephropathy with Tripterygium wilfordii Hook F extract: a prospective, 
randomized, controlled clinical trial," Journal of Translational Medicine, vol. 11, no. 1, p. 134, 2013.

[63] Y. Zhong, M. C. Menon, Y. Deng, Y. Chen, and J. C. He, "Recent advances in traditional Chinese medicine for kidney disease," American Journal of Kidney Diseases, vol. 66, no. 3, pp. 513-522, 2015.

[64] X. L. Xu, L. J. Yang, and J. G. Jiang, "Renal toxic ingredients and their toxicology from traditional Chinese medicine," Expert Opinion on Drug Metabolism \& Toxicology, vol. 12, no. 2, pp. 149-159, 2016. 